\title{
Toward cancer treatment using terahertz radiation: demethylation of cancer cells
}

Joo-Hiuk Son, Hwayeong Cheon

Joo-Hiuk Son, Hwayeong Cheon, "Toward cancer treatment using terahertz radiation: demethylation of cancer cells," Proc. SPIE 11390, Next-Generation Spectroscopic Technologies XIII, 1139002 (24 April 2020); doi: 10.1117/12.2557655

SPIE. Event: SPIE Defense + Commercial Sensing, 2020, Online Only 


\title{
Toward cancer treatment using terahertz radiation: Demethylation of cancer cells
}

\author{
Joo-Hiuk Son ${ }^{1,2, *}$ and Hwayeong Cheon ${ }^{1}$ \\ ${ }^{1}$ University of Seoul, Seoul 02504, Republic of Korea \\ ${ }^{2}$ iNexus, Inc., Gyeonggi-do, Republic of Korea \\ *Email: joohiuk@uos.ac.kr
}

\begin{abstract}
Carcinogenesis involves DNA methylation, a primary alteration in DNA, in the development of cancer before genetic mutation. Because abnormal DNA methylation is found in most cancer cells, the assessment and manipulation of DNA methylation using terahertz radiation can be a novel optical method to detect and control cancer. By directly observing the methylation via terahertz spectroscopy at approximately $1.65 \mathrm{THz}$, this epigenetic chemical change can be manipulated to the state of demethylation using high-power terahertz radiation. Demethylation of cancer DNA is a key issue in epigenetic cancer therapy, and our results may lead to the treatment of cancer using electromagnetic waves.
\end{abstract}

Keywords: Terahertz, biomedical, cancer diagnosis, cancer therapy, methylation

\section{INTRODUCTION}

$\mathrm{T}$ ERAHERTZ electromagnetic waves, whose spectrum lies between microwave and infrared regions (0.1-10 THz), have been utilized for the diagnosis and imaging of cancer [1-3]. In a bid to find a specific signal for such measurements, a terahertz resonance fingerprint of cancer has been directly observed at $1.65 \mathrm{THz}$ for several types of cancer [4]. The resonant signal is believed to originate from aberrant methylation in DNA, which is an epigenetic modification prior to genetic mutation in the development of cancer. If it is a true resonant feature, this might be controlled or manipulated using a terahertz radiation at the frequency.

In this paper, the details of finding the terahertz resonance of cancer DNA coming from methylation and the manipulation to demethylation using resonant terahertz radiation will be explained. In addition, the relation of demethylation to cancer treatment will be discussed.

\section{RESULTS}

Because the abnormal regulation of gene expression can cause carcinogenesis, DNA methylation, which plays a role in the regulation of gene expression, is a critical factor in cancer research and treatment. The terahertz characteristics of methylated cytidine, a nucleoside, provided the clues to the resonance fingerprint of DNA methylation (see the inset of Fig. 1). In aqueous solutions, we tracked and observed the molecular resonance of genomic DNA from two controls (293T, M-293T) and five types of cancer (PC3 - prostate cancer, A431 - skin cancer, A549 - lung cancer, MCF-7 - breast cancer, SNU-1 - gastric cancer) cell lines, using freezing technique and baseline correction, as shown in Fig. 1 [4]. The amplitudes of the resonance signals were dependent on the types of cancer cells the DNA had come from. These signals were quantified in order to identify cancer cell types, and the results were similar to those of ELISA reaction, a biological quantification method [4].

The resonance peak of the spectrum not only presented the existence and quantity of methylation in DNA, but also the target for manipulation of DNA methylation to control the gene expression in cancer DNA by breaking the methyl bond. To reduce the degree of methylation, we irradiated a resonant high-power terahertz radiation, which is generated from a $\mathrm{LiNbO}_{3}$ crystal driven by $1-\mathrm{kHz}$ regenerative amplifier and has a limited bandwidth around the resonance frequency of 
DNA methylation by a bandpass filter, onto cancer DNA. One of the results is shown in Fig. 2. The DNA from human embryonic kidney cell line (293T) was utilized as a control and a part of it was artificially methylated to give M-293T using DNA methyltransferase enzyme (DNMT). The M-293T was further divided into two, of which one was irradiated with high-power terahertz radiation. As can be seen in Fig. 2, the methylation level of M-293T was high but it was decreased by half, close to the level of 293T, after the exposure. The terahertz results were also cross-checked using ELISA reaction method, as shown in Fig. 3 [4].

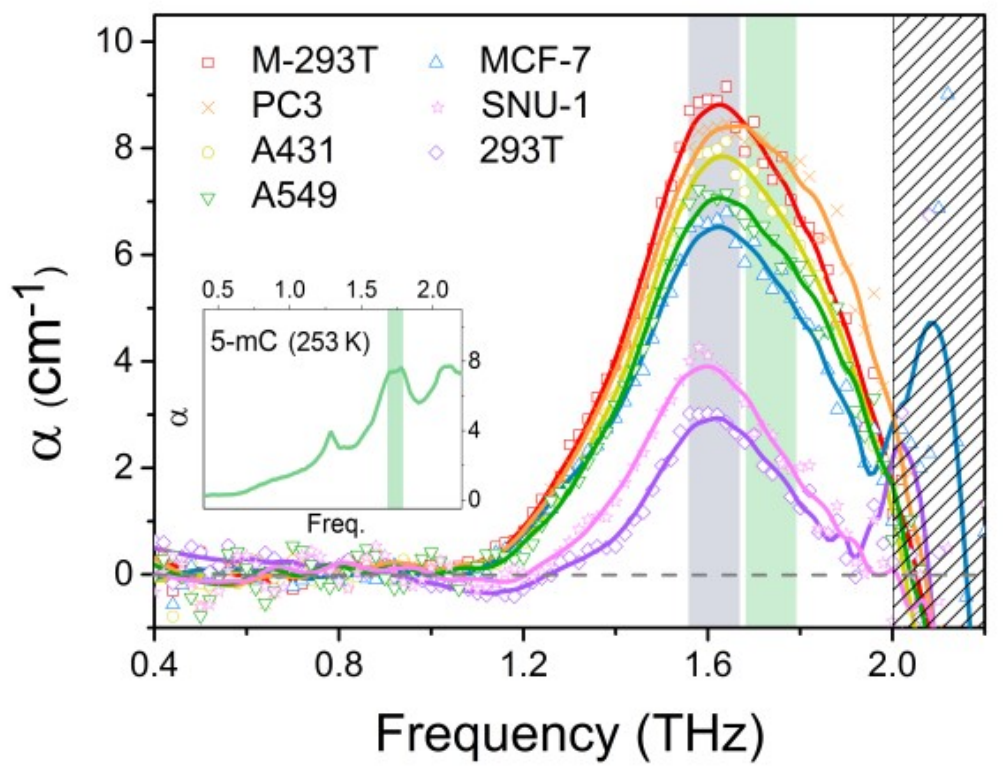

Fig. 1. Resonance fingerprints of normal and cancer DNAs [4].

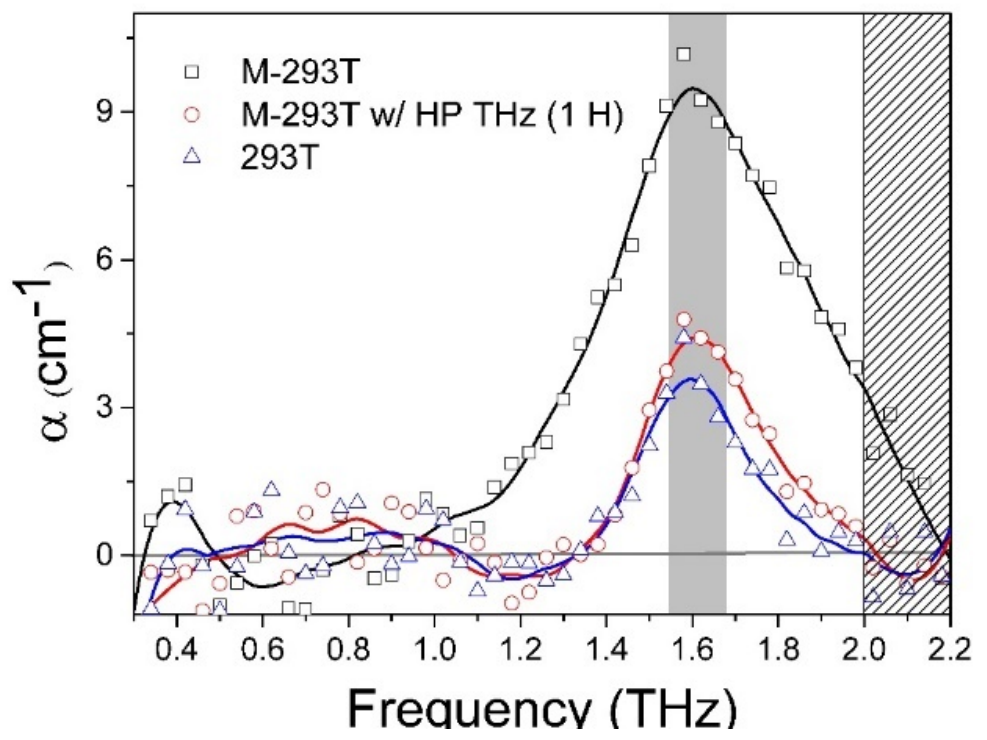

Fig. 2. Demethylation of DNA after the exposure to high-power terahertz radiation [5]. 


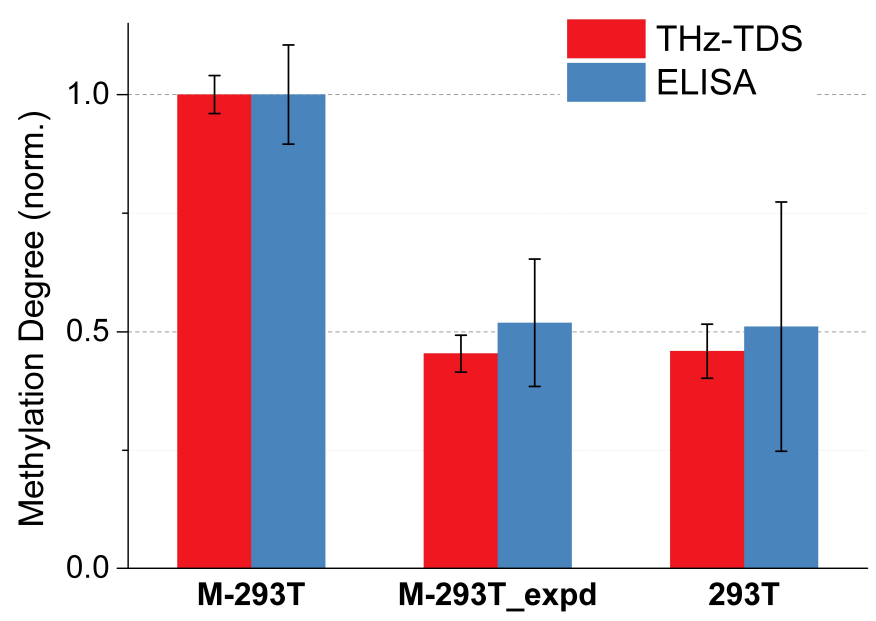

Fig. 3. Verification of terahertz demethylation of DNA using ELISA reaction [5].

To assess the demethylation effect in actual cancer DNA, we applied high-power terahertz radiation on some types of blood cancer DNA. The genomic DNA of blood cancer cell lines had five types: T-cell lymphoma (SU-DHL1), B-cell lymphoma (SU-DHL9 and OCI-LY1), Burkitt lymphoma (Raji), T-cell acute lymphoblastic leukemia (T-ALL, CCRFCEM and Jurkat), and acute myeloid leukemia (AML, HL-60). The blood cancer DNA experiment was performed using the same method with the M-293T. As can be found in Fig. 4, the degree of DNA methylation was significantly decreased in most blood cancers, although the demethylation ratios varied according to the cell line (approximately 10 $70 \%)[5]$.
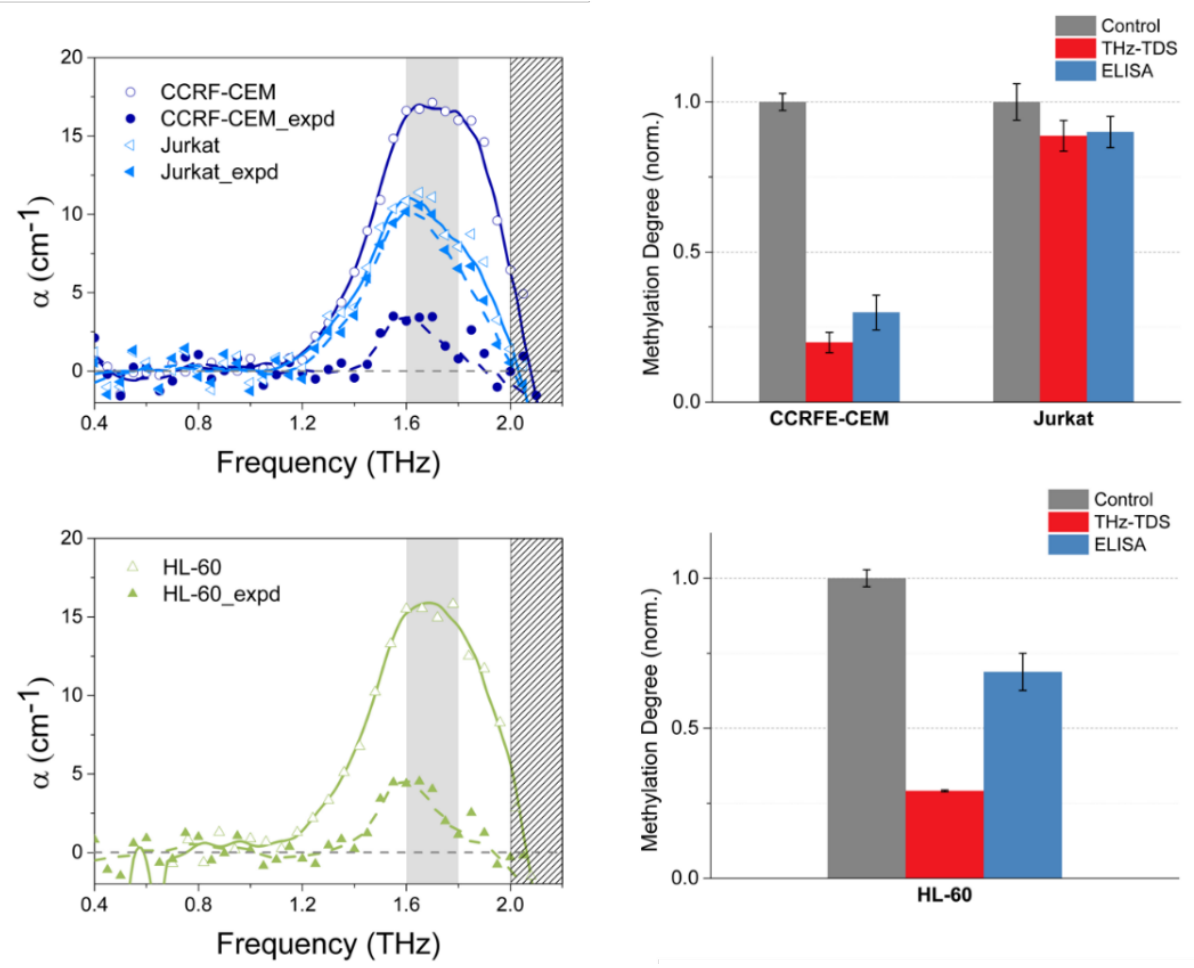

(a) 

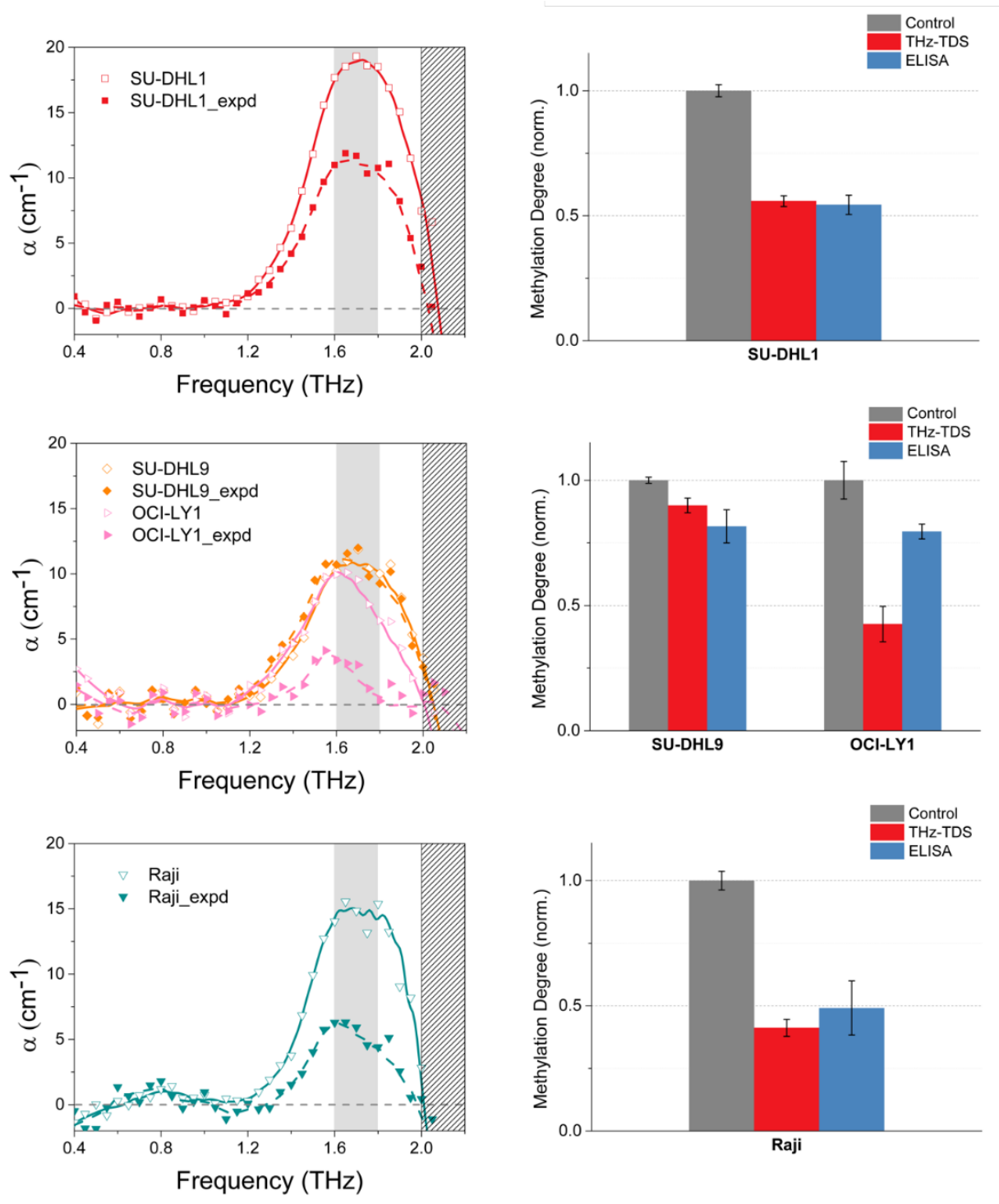

(b)

Fig. 4. Demethylation of (a) leukemia and (b) lymphoma DNA using the resonant high-power terahertz radiation [5].

Solid cancers such as breast cancer (MCF7) also showed its resonant peak around $1.6 \mathrm{THz}$, which was decreased to approximately half amplitude by high-power terahertz radiation, as shown in Fig. 5. This implies that resonant terahertz radiation can also induce the demethylation of solid cancer DNA [2].

The effective demethylation of melanoma cells, without extracting the cancer DNA, was also achieved using a resonant terahertz radiation, as shown in Fig. 6 [6]. The degree of demethylation was approximately 15\%, which occurs in natural cell structure. 

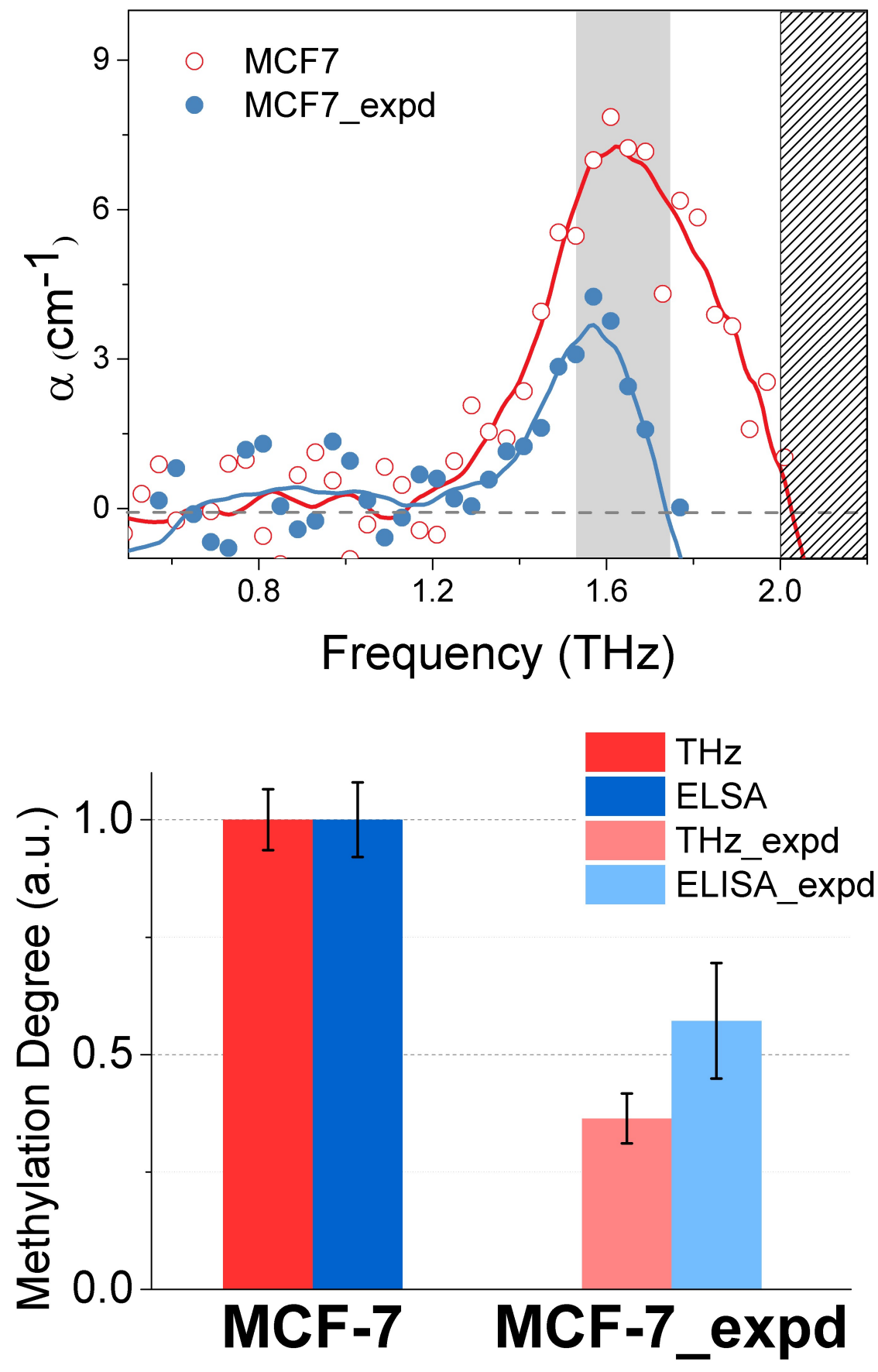

Fig. 5. Demethylation of (a) breast cancer (MCF7) DNA using the resonant high-power terahertz radiation and (b) the THz results agree with that of ELISA quantification [2]. 


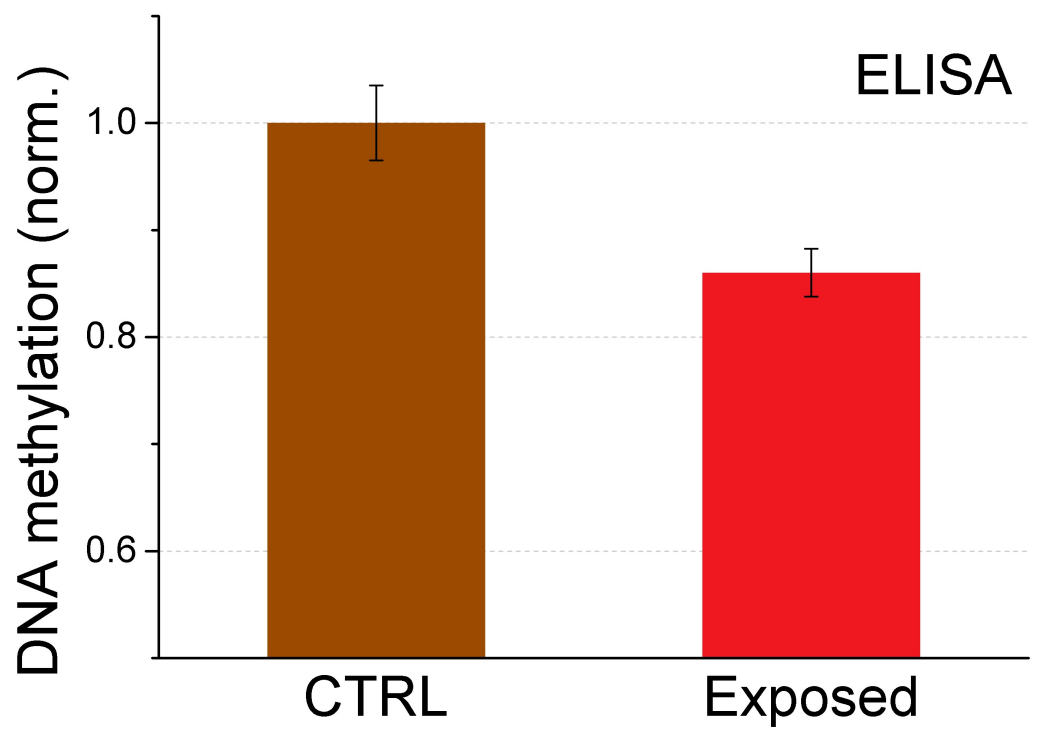

Fig. 6. Demethylation of melanoma cells (SK-MEL-3) using the resonant high-power terahertz radiation [6].

\section{SUMMARY}

The results demonstrate that the molecular resonance of cancer DNA exists in the terahertz region, and this can be controlled by the irradiation of high-power terahertz radiation. This is the first result for manipulating DNA methylation using an optical technique. The manipulation of methylation in cancer DNA is an important issue in epigenetic cancer therapy because aberrant DNA methylation can lead to abnormal gene expression. Although there are several chemical inhibitor drugs for DNA demethylation, which reduce aberrant DNA methylation in cancer cells, they still have a high risk of side effects. Because our method is a non-invasive, non-ionizing, and non-labelling technique, and uses specific resonance frequency, it might offer an effective solution to achieve a novel cancer therapy with few side effects. This implies that, like genetic scissors in biology, terahertz technique may be applied as "epigenetic scissors," which could lead to the demethylation of cancer DNA.

\section{REFERENCES}

[1] J.-H. Son, ed., Terahertz Biomedical Science and Technology, 1st ed., Boca Raton, FL, USA: CRC (2014).

[2] J.-H. Son, et al., "Potential clinical applications of terahertz radiation," J. Appl. Phys., vol. 125, 190901 (2019).

[3] H. Cheon, et al., "Toward clinical cancer imaging using terahertz spectroscopy," IEEE Sel. Top. Quant. Elec., vol. 23, 8600109 (2017).

[4] H. Cheon, et al., "Terahertz molecular resonance of cancer DNA," Sci. Rep., vol. 6, 2016, Art. no. 37103 (2016).

[5] H. Cheon, et al., "Detection and manipulation of methylation in blood cancer DNA using terahertz radiation," Sci. Rep., vol. 9, Art. no. 6413 (2019).

[6] H. Cheon, et al., "Effective demethylation of melanoma cells using terahertz radiation," Biomed. Opt. Exp., vol. 10, 4931 (2019). 